\title{
Design variations in vertical muscle-sparing thoracotomy
}

\author{
Noriaki Sakakura, Tetsuya Mizuno, Takaaki Arimura, Hiroaki Kuroda, Yukinori Sakao \\ Department of Thoracic Surgery, Aichi Cancer Center Hospital, Nagoya, Japan \\ Correspondence to: Noriaki Sakakura. Department of Thoracic Surgery, Aichi Cancer Center Hospital, 1-1 Kanokoden, Chikusa-ku, Nagoya 464- \\ 8681, Japan. Email: nsakakura@aichi-cc.jp.
}

Submitted Mar 22, 2018. Accepted for publication Jul 03, 2018.

doi: 10.21037/jtd.2018.07.100

View this article at: http://dx.doi.org/10.21037/jtd.2018.07.100

\section{Introduction}

Open thoracotomy continues to be the gold standard approach for thoracic surgery despite the recent developments in thoracoscopic and robot-assisted procedures. Vertical muscle-sparing thoracotomy (VMST) is a well-established technique (1-3) that has not attracted much attention in recent years. Surgeons often regard VMST as an anterior approach; however, it actually allows wide access to even the ventral and dorsal aspects of the thorax. In this paper, we discuss the variations in VMST, mainly with respect to the incision design, which have not been fully discussed to date. By its optimal incision design, VMST balances optimal skeletal muscle preservation with favorable exposure of various thoracic structures, including the dorsal aspect. We enumerate these VMST variations used in our practice, briefly discuss the basic but important procedures, and hope that our report will assist general thoracic surgeons, particularly the young trainees and residents, in advancing their clinical skills.

\section{Surgical technique}

The standard VMST technique has been demonstrated by Ginsberg (1) and Hennington and colleagues (2). Briefly, after a vertical incision is made along the midaxillary line, the latissimus dorsi (LD) muscle is retracted posteriorly and the posterior border of the serratus anterior (SA) muscle is mobilized anteriorly to expose the thorax. By the procedure of sparing LD and SA muscles, the thoracodorsal neurovascular bundle and long thoracic nerve are naturally preserved.

Surgeons often regard VMST as an anterior approach owing to its similarity with anterolateral thoracotomy in terms of sparing or splitting of the SA muscle. However,
VMST actually allows wide access to the ventral and dorsal aspects of the thorax depending on the incision location. Design variations that we use in practice are illustrated by lines A-H in Figure 1:

(A) This incision line provides good access to the ventral thorax; however, when the incision is small, it limits access to the dorsal aspect of the hilum.

(B) This represents an extended incision line positioned posterior to line A.

(C) This vertical axillary line represents a typical VMST incision, which ensures optimal access to both the ventral and dorsal aspects of the hilum.

(D) This vertical incision, dorsal to line C, provides better access to the posterior aspect of the thoracic structure. Although small, this incision provides access to both the ventral and dorsal aspects of the hilum.

(E,F) These incision lines along the anterior border of the LD muscle allow good access to the dorsal aspect of the hilum.

$(\mathrm{G}, \mathrm{H})$ These lines represent the incisions along the middle region of the LD muscle. Sparing or splitting this muscle allows better exposure of the posterior aspect of the thorax compared with that allowed by lines $\mathrm{E}$ and $\mathrm{F}$.

Surgeons can control the width of the thoracotomy based on the location of the incision, length of the cranial to caudal incision, and extent of the intercostal dissection. Although any larger vertical incision may allow a wider range of thoracic structures to be exposed, the variations described here can be established through sparing or splitting both the SA and LD muscles in various directions to achieve optimum exposure even with a relatively small 


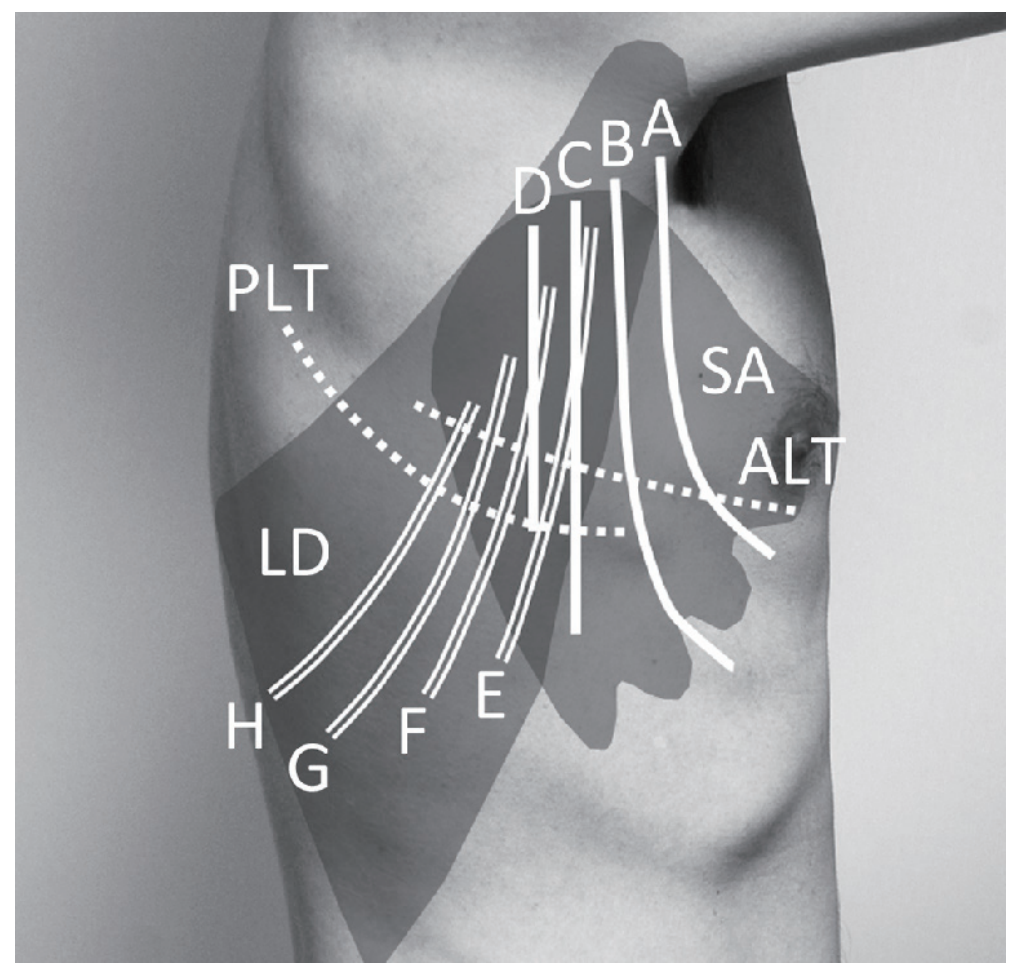

Figure 1 Variations in incision designs for vertical muscle-sparing thoracotomy (lines A-H). ALT, anterolateral thoracotomy; LD, latissimus dorsi muscle; PLT, posterolateral thoracotomy; SA, serratus anterior muscle.

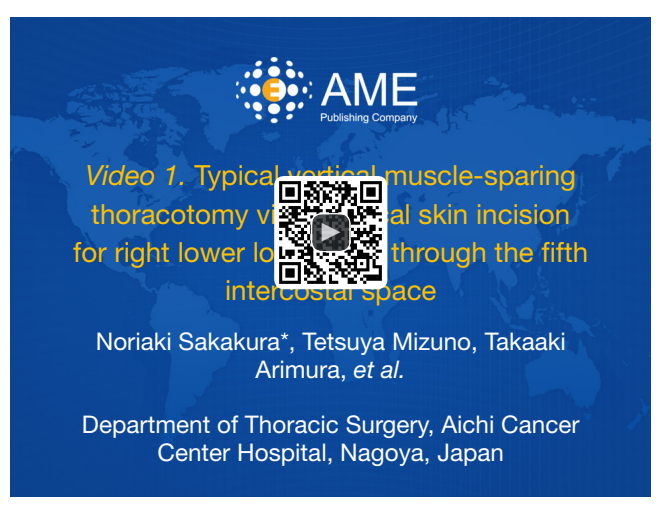

Figure 2 Typical vertical muscle-sparing thoracotomy via a vertical skin incision for right lower lobectomy through the fifth intercostal space (4). The incision design is made along line $\mathrm{C}$, as illustrated in Figure 1. The latissimus dorsi (LD) muscle is retracted posteriorly, and the posterior border of the serratus anterior (SA) muscle is mobilized anteriorly to expose the thorax. The LD and SA muscles were completely preserved and the thoracodorsal neurovascular bundle and long thoracic nerve are naturally preserved. This approach also allows optimal access to both the ventral and dorsal aspects of the hilum.

Available online: http://www.asvide.com/article/view/26628

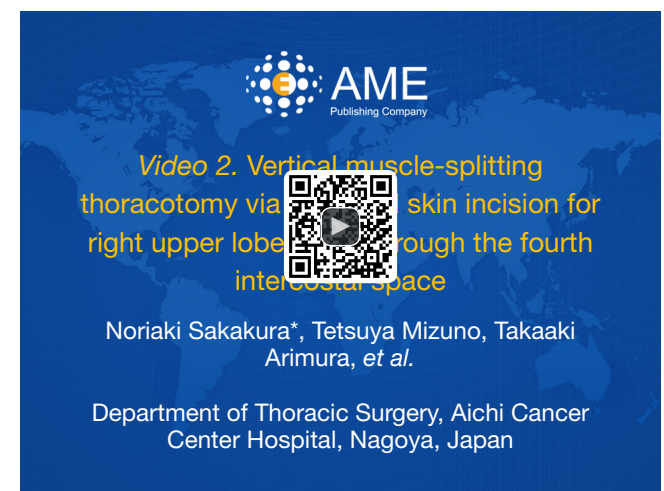

Figure 3 Vertical muscle-splitting thoracotomy via a vertical skin incision for right upper lobectomy through the fourth intercostal space (5). The incision design is made along line $\mathrm{D}$, as illustrated in Figure 1. The latissimus dorsi (LD) muscle is spared, and the serratus anterior (SA) muscle is split to ensure optimal access to both the ventral and dorsal aspects of the hilum even with a relatively small incision.

Available online: http://www.asvide.com/article/view/26629 

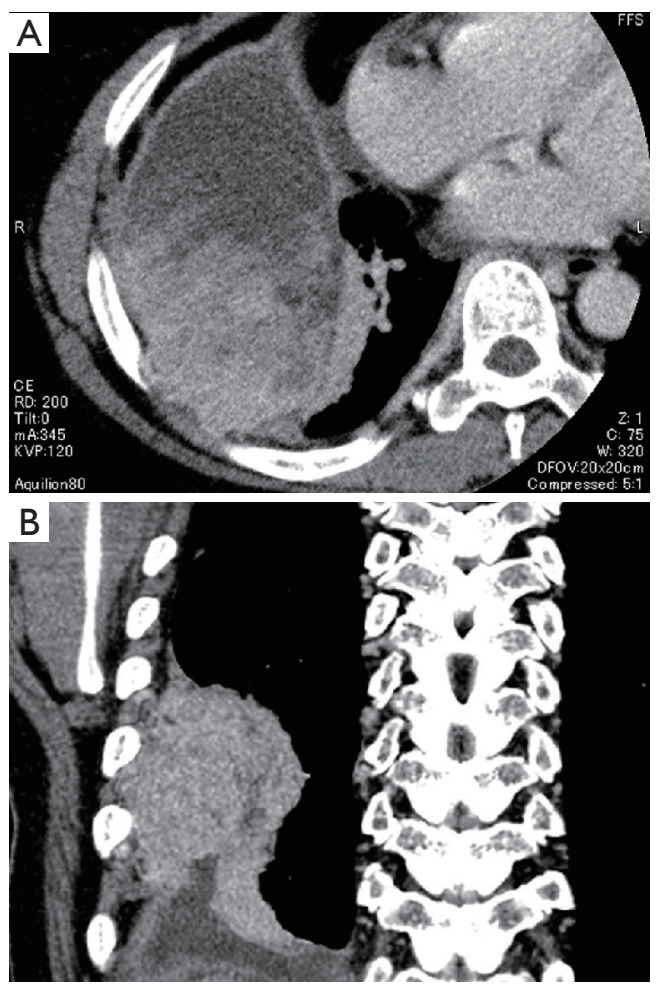

Figure 4 Radiological findings of the tumor originating from the dorsal chest wall (A, axial view; B, coronal view).

incision.

Here, we present brief videos of two typical thoracotomy procedures of "sparing" (Figure 2) and "splitting" (Figure 3) the SA muscle via a vertical skin incision that we perform in our daily clinical practice.

As an example of a skin incision variant, we present the case of a 58-year-old woman with spindle-cell sarcoma that originated in the dorsal portion of the intrathoracic chest wall and infiltrated the seventh and eighth ribs (Figure 4). Because the patient was a professional dancer, she strongly desired that her LD and other skeletal muscles be preserved. Therefore, we adopted an alternative VMST to mobilize and retract the LD muscle posteriorly (Figure $5 A$ ). The tumor and involved ribs were resected (Figure $5 B$ ); the chest wall defect was reconstructed; and the LD muscle was restored to its original position (Figure $5 C$ ). The incision design (Figure 5D) was made along line G, as illustrated in Figure 1. Although in this case, many surgeons would have selected a conventional posterolateral thoracotomy (PLT) approach, which involves cutting the LD muscle, this variant of VMST can be an alternative choice due to its less invasive nature.

\section{Discussion}

Noteworthy articles on VMST were published in the 1980s and 1990s (1-3). Prominent surgeons reported that compared with PLT, VMST is less invasive, helps achieve better postoperative pulmonary and shoulder functions, and yields superior cosmetic results. Instead of retracting the posterior border of the SA muscle, a similar procedure of splitting the muscle has previously been reported (6). Others have insisted that the VMST approach cannot ensure adequate exposure of the thoracic cavity and proposed a different procedure that involves an alternative PLT and splitting and preservation of the LD muscle (7).

Although VMST is generally considered superior to conventional lateral thoracotomy from the viewpoint of postoperative pain, pulmonary function, or shoulder function (1-3), clinical results reported were not necessarily consistent. For example, Ponn and coworkers reported that limited incisions may result in slightly better late pulmonary function, but the differences are small and of no apparent clinical advantage in an average patient (8). Landreneau and colleagues also described that the relative efficacies and occurrence of acute or chronic morbidity are almost equivalent between the two types of thoracotomy, and the advantage of muscle-sparing thoracotomy involves the preservation of chest wall musculature in case rotational muscle flaps are needed (9). Recently, a meta-analysis in which 12 trials were included, comprising 571 patients in the muscle-sparing thoracotomy group and 512 patients in the PLT group, showed that muscle-sparing procedures resulted in quicker postoperative recovery and better shoulder function (10).

Readers may consider open thoracotomy to be so extensively studied that no additional evaluation is required. However, even in this era of novel thoracoscopic and robotic surgeries, the importance of open procedures in routine medical practice cannot be emphasized enough. VMST is considered a basic but important technique for reducing the invasiveness of open procedures, and its practicality should be repeatedly recognized, regardless of how often we use it. Although VMST procedure could be performed in a better way using optimal skin incision designs, as illustrated in Figure 1, these alternative incisions have rarely been presented to date. We would like to emphasize this point in this brief report.

VMST incision, which is made perpendicular to the PLT incision, offers several advantages such as avoiding the dissection of subcutaneous tissues above the LD muscle 

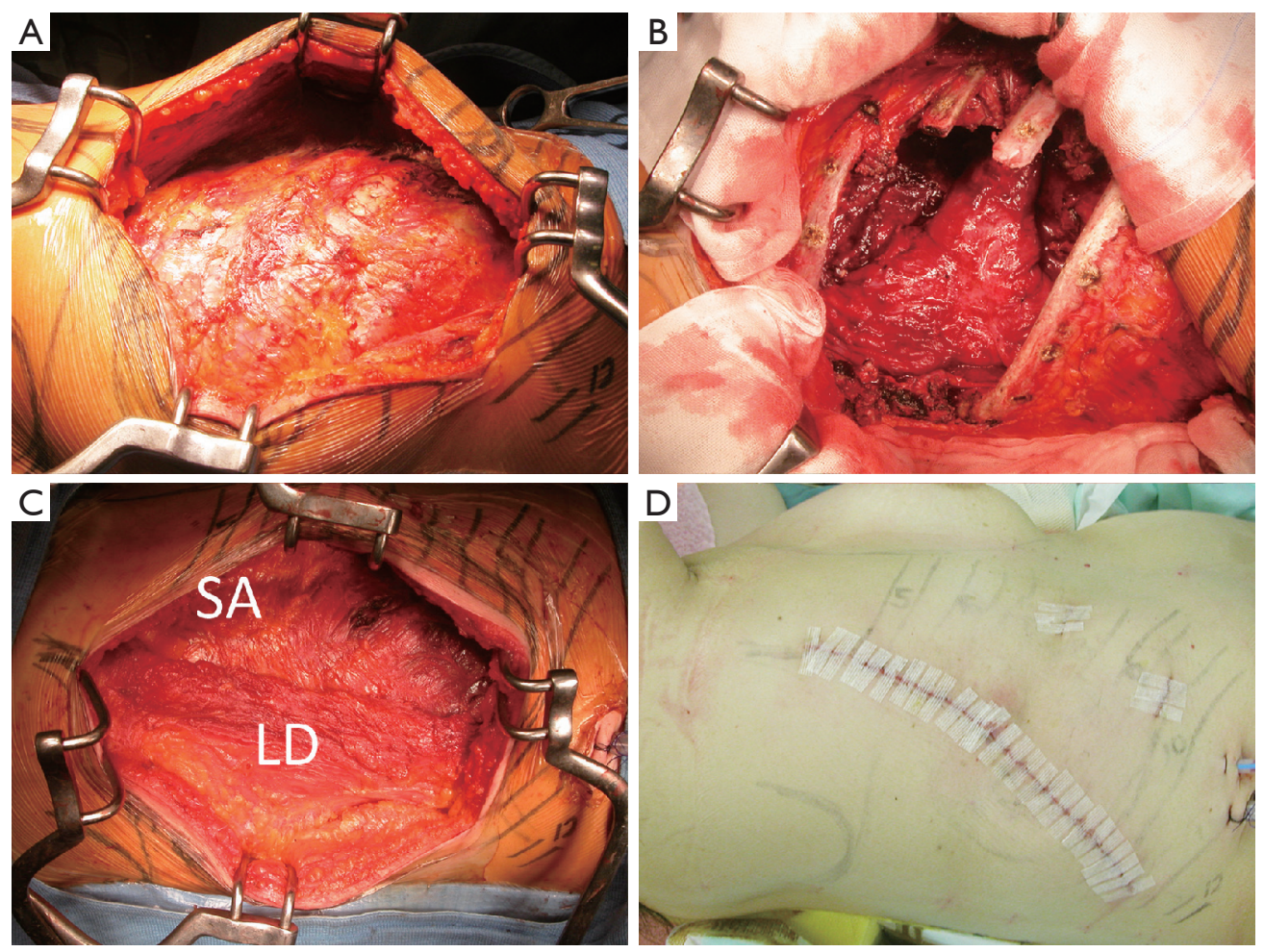

Figure 5 Intraoperative views of an alternative vertical muscle-sparing thoracotomy. (A) The latissimus dorsi (LD) muscle is retracted posteriorly, and the posterior border of the serratus anterior (SA) muscle is mobilized anteriorly to expose the thorax; (B) the tumor and involved ribs were resected; (C) the LD and SA muscles were restored to its original position; (D) the incision design was made along line G, as illustrated in Figure 1. Detailed technical aspects are described in the text.

fascia. Preservation of the LD muscle is also important because its flaps can be used in a variety of surgical treatments for vital conditions $(8,11)$. On the contrary, VMST may involve some disadvantages with regard to clinical and cosmetic perspectives because the chosen incision lines may not be parallel to Langer's cleavage lines. A relatively wider dissection can predispose an individual to develop a subcutaneous seroma (10); therefore, suturing the skeletal muscle fascia to the subcutaneous tissue of the chest wall and postoperative use of compression garments are recommended as preventive measures.

At our institution, these VMST variants have been adopted even for subcarinal node dissection and esophageal cancer surgeries. In such cases, appropriate retraction of the bronchus and other hilar structures is mandatory to facilitate optimal view. Although based on practical and educational perspectives, we commonly apply conventional PLT for patients that require subcarinal node dissection, particularly on the left, the VMST variants can also be suitable choices for patients who need skeletal muscle preservation.

However, VMST provides limited access to superior sulcus lesions. The choice of the most appropriate incision among various options, such as the hook, hemi-clamshell, open-door, or transmanubrial approaches, depends on the surgery. As a reference, the number of the thoracotomy types performed between January 2013 to December 2017 in our department were approximately the following: vertical axillary, 305 cases; posterolateral, 330 cases; hook approach, 9 cases; hemi-clamshell, 2 cases; median sternotomy, 37 cases; thoracoscopic, 1,120 cases; other alternative, 6 cases.

A surgery with a wide selection of approaches and a delicate technique leads to better outcomes. Via its optimal incision design, VMST balances optimal skeletal muscle preservation with favorable exposure of various thoracic structures, including the dorsal aspect. Although this brief report does not propose a novel surgical technique, we hope that it will assist thoracic trainees and residents in improving their clinical skills. 


\section{Acknowledgements}

Dr. Syunzo Hatooka and his coworkers developed and established the basic procedural aspect of VMST at our institution, which we have modified.

\section{Footnote}

Conflicts of Interest: The authors have no conflicts of interest to declare.

Informed Consent: Each patient was informed that his or her clinical data could be used for various clinical studies, and written informed consent for this report was obtained on this basis.

\section{References}

1. Ginsberg RJ. Alternative (muscle-sparing) incisions in thoracic surgery. Ann Thorac Surg 1993;56:752-4.

2. Hennington MH, Ulicny KS Jr, Detterbeck FC. Vertical muscle-sparing thoracotomy. Ann Thorac Surg 1994;57:759-61.

3. Pochettino A, Bavaria JE. Anterior axillary muscle-sparing thoracotomy for lung transplantation. Ann Thorac Surg 1997;64:1846-8.

4. Sakakura N, Mizuno T, Arimura T, et al. Typical vertical muscle-sparing thoracotomy via a vertical skin incision for

Cite this article as: Sakakura N, Mizuno T, Arimura T, Kuroda H, Sakao Y. Design variations in vertical musclesparing thoracotomy. J Thorac Dis 2018;10(8):5115-5119. doi: 10.21037/jtd.2018.07.100 right lower lobectomy through the fifth intercostal space. Asvide 2018;5:710. Available online: http://www.asvide. com/article/view/26628

5. Sakakura N, Mizuno T, Arimura T, et al. Vertical musclesplitting thoracotomy via a vertical skin incision for right upper lobectomy through the fourth intercostal space. Asvide 2018;5:711. Available online: http://www.asvide. com/article/view/26629

6. Siegel T, Steiger Z. Axillary thoracotomy. Surg Gynecol Obstet 1982;155:725-7.

7. Subramanian S, Halow KD. Muscle-splitting posterolateral thoracotomy: a novel technique. Curr Surg 2000;57:74-7.

8. Ponn RB, Ferneini A, D'Agostino RS, et al. Comparison of late pulmonary function after posterolateral and musclesparing thoracotomy. Ann Thorac Surg 1992;53:675-9.

9. Landreneau RJ, Pigula F, Luketich JD, et al. Acute and chronic morbidity differences between muscle-sparing and standard lateral thoracotomies. J Thorac Cardiovasc Surg 1996;112:1346-50; discussion 1350-1.

10. Uzzaman MM, Robb JD, Mhandu PC, et al. A metaanalysis comparing muscle-sparing and posterolateral thoracotomy. Ann Thorac Surg 2014;97:1093-102.

11. Sakakura N, Mizuno T, Kuroda H, et al. Surgical treatment of empyema after pulmonary resection using pedicle skeletal muscle plombage, thoracoplasty, and continuous cavity ablution procedures: a report on three cases. J Thorac Dis 2016;8:1333-9. 